\title{
DETECÇÃO DE Heterodera glycines EM PLANTIO DE SOJA MEDIANTE ESPECTRORRADIOMETRIA NO VISÍVEL E INFRAVERMELHO PRÓXIMO*
}

\author{
RENATO F. DOS SANTOS JUNIOR ${ }^{1 * *}$, JAIME M. DOS SANTOS ${ }^{1}$, \\ BERNARDO F. T. RUDORFF' ${ }^{2} \&$ IVO A. MARCHIORATO ${ }^{1}$
}

\begin{abstract}
'Departamento de Fitossanidade, Universidade Estadual Paulista, Faculdade de Ciências Agrárias e Veterinárias, Campus de Jaboticabal, CEP 14884-900, Jaboticabal, SP, e-mail: fchapada@ hotmail.com; ${ }^{2}$ Instituto Nacional de Pesquisas

Espaciais - INPE, Av. dos Astronautas, 1.758, CEP 12227-010, São José dos Campos, SP
\end{abstract}

(Aceito para publicação em 13/11/2001)

Autor para correspondência: Renato F. dos Santos Jr.

SANTOS JUNIOR., R.F., SANTOS, J.M., RUDORFF, B.F.T. \& MARCHIORATO, I.A. Detecção de Heterodera glycines em plantio de soja mediante espectrorradiometria no visível e infravermelho próximo. Fitopatologia Brasileira 27:355-360. 2002.

\section{RESUMO}

$\mathrm{O}$ ataque do nematóide de cisto da soja, Heterodera glycines, limita o potencial de expansão e maior produtividade de áreas plantadas com soja (Glycine Max). O conhecimento da distribuição espacial desse patógeno na lavoura é fundamental, para elaboração de estratégias de manejo. A área em estudo estava localizada em lavoura de soja, variedade BRS133, localizada no Município de Florínea, SP, com solos naturalmente infestados por H. glycines. Foram obtidas medidas de espectrorradiometria de campo, 112 dias após o plantio, nas regiões do visível e do infravermelho próximo do espectro eletromagnético, a fim de se conhecer o padrão da resposta espectral de plantas atacadas pelo fitonematóide. Paralelamente, foram retiradas amostras de solo e encaminhadas ao Laboratório de Nematologia, Departamento de Fitossanidade da Universidade Estadual Paulista Júlio de Mesquita Filho, Campus de Jaboticabal, onde foram processadas para determinação da densidade populacional do nematóide. As medidas do espectrorradiômetro foram transformadas em índice vegetativo, com diferença normalizada (NDVI), que foi relacionado com a densidade populacional do nematóide, peso da matéria fresca e número de vagens por planta. Observou-se que diferentes densidades de população estão diretamente relacionados com a resposta espectral das plantas expressa, através dos valores do NDVI.

Palavras-chave adicionais: Sensoriamento remoto, espectrorradiometria de campo, resposta espectral, fitonematóides.

\section{ABSTRACT \\ Detection of infested areas with Heterodera glycines in a soybean field using spectroradiometry in the visible and near infrared}

The damage caused by the soybean cyst nematode, Heterodera glycines, not only limits expansion of the soybean (Glycine Max) crop but also reduces the yield of soybeans in Brazil. Knowledge of the spatial distribution of this pathogen is needed in order to establish management measures. This study was conducted in Florínea County, São Paulo, Brazil, in a commercial soybean field, cultivar BRS133, previously known to be infected with $\mathrm{H}$. glycines. In order to see the pattern of the spectral response of plants attacked by the phytonematode, field spectroradiometry measurements in the visible and near infrared regions of the electromagnetic spectrum were obtained 112 days after sowing. At the same time, soil samples were taken to the "Laboratório de Nematologia do Departamento de Fitossanidade da Universidade Estadual Paulista Júlio de Mesquita Filho, Campus de Jaboticabal", where they were processed and where the population density of the nematode was determined. The spectroradiometric measurements were transformed to the normalized difference vegetation index (NDVI) that was related to population density of the nematode, to fresh biomass and to pods per plant. It was observed that different population densities are directly related to the spectral response of the plants expressed by NDVI values.

\section{INTRODUÇÃO}

A soja [Glycine max (L.) Merr.] é o mais importante produto agrícola na pauta das exportações brasileiras, com receita anual em torno de cinco bilhões de dólares. O Brasil é o segundo maior produtor dessa oleaginosa no mundo, sendo o País com maior potencial de expansão das áreas plantadas

\footnotetext{
*Parte da Dissertação de Mestrado do primeiro autor. Universidade Estadual Paulista, Faculdade de Ciências Agrárias e Veterinárias, Campus de Jaboticabal.
}

**Bolsista da FAPESP e crescente aumento de produtividade (PESQUISA FAPESP, 2001). O nematóide de cisto da soja (NCS), Heterodera glycines Ichinohe, constitui grave e limitante fator desse potencial.

Os sintomas observados com o ataque de $H$. glycines no campo são reboleiras de plantas com porte reduzido, folhas cloróticas, abortamento de flores e vagens, deterioração das raízes e haste verde ao final do ciclo. A intensidade dos sintomas depende da população do fitonematóide e fertilidade do solo (Yorinori, 1997).

O mapeamento de áreas infestadas por fitonematóides 


\section{R.F. Santos Júnior et al.}

é importante instrumento de apoio às decisões sobre manejo de populações. O método tradicional de amostragem no campo, em áreas extensas como são as plantadas com soja, requer coleta de grande número de amostras de solo que não refletem com precisão a distribuição espacial do nematóide.

As plantas emitem e refletem radiação eletromagnética, que pode ser captada por sensores especiais sensíveis a determinados comprimentos de onda. Essa radiação fornece informações sobre desenvolvimento e sanidade das culturas e permite acompanhamento (Lamparelli et al. 2001).

O uso de técnicas de sensoriamento remoto para identificar e mapear áreas infestadas por fitonematóides pode ser instrumento viável no manejo. Estudos detalhados sobre variação da resposta espectral de culturas agrícolas através de técnicas de sensoriamento remoto podem ser realizados utilizando-se espectrorradiometria de campo, com a qual se obtém medidas de reflectância da cultura a poucos metros acima do dossel (2 a $5 \mathrm{~m}$ ) em várias faixas de comprimento de onda geralmente compreendidas nas regiões do visível e do infravermelho próximo do espectro eletromagnético (Batista \& Rudorff, 1990, Rudorff et al., 1997).

Na região do visível (400 a $700 \mathrm{~nm}$ ), a presença de pigmentos clorofilados é responsável pela maior absorção da radiação nas porções azul e vermelho, sendo menos absorvida na porção do verde (Knipling, 1970), ou seja, plantas verdes e sadias refletem pouca energia nos comprimentos de onda correspondentes ao azul e vermelho. No infravermelho próximo (760 a $1300 \mathrm{~nm}$ ), a estrutura interna das folhas exerce grande influência sobre o espectro, nessa faixa a radiação é fortemente espalhada sofrendo múltiplas reflexões e transmissões ao incidir na vegetação, de tal forma que quanto maior for o índice de área foliar maior será a energia refletida (Myers, 1970, Amaral et al., 1990).

A quantidade de energia refletida pelo dossel da cultura, nos comprimentos de onda correspondentes ao vermelho e infravermelho próximo, é significativamente correlacionada com a atividade fotossintética das plantas (Holben et al., 1980, Daughtry et al., 1984). Plantas afetadas por doenças geralmente apresentam crescimento reduzido e sintomas de amarelecimento, que proporcionam contraste na resposta espectral em relação às saudáveis (Brenchley, 1968).

Através dos dados obtidos em leituras radiométricas, são calculados Índices de Vegetação, que são parâmetros aplicados à identificação de variabilidade na cultura. Valeriano (1992), considerou a importância do índice de área foliar para estimativa de biomassa verde e estudou com detalhes o comportamento espectral do trigo [Triticum aestivum (L.) Thell.], na região dos cerrados. Essa constatação foi corroborada por Formaggio (1985). Segundo esse autor, quando a cobertura de solo é completa, as folhas são elementos dominantes nas plantas, quanto ao aspecto das influências sobre as propriedades espectrais de dosséis vegetais.

Em estudos realizados utilizando-se sensoriamento remoto na agricultura, tem sido comum adoção do Índice Vegetativo com Diferença Normalizada (NDVI - Normalised Difference Vegetation Index). Taylor et al. (1997) utilizando- se desse parâmetro na estimativa de produtividade em lavouras de milho (Zea mays L.), constataram boas correlações com o peso de grãos por unidade de área. QI et al. (1994), testaram diversos índices obtidos com sensoriamento remoto não orbital, quanto a caracterização da variação temporal e espacial da vegetação, visando restringir as influências do solo. Nesse sentido, em condição de cobertura vegetal total, o NDVI, em relação a outros índices, apresentou melhor desempenho, minimizando comparativamente efeitos da influência do solo.

Recursos possibilitando utilização de técnicas de sensoriamento remoto para grandes áreas, tendem a tornarse cada vez mais acessíveis com o avanço tecnológico e disponibilização de novos satélites com maior resolução espacial e alta freqüência temporal. O objetivo desse estudo foi verificar a viabilidade da utilização de medidas de espectrorradiometria de campo, nas regiões do visível e do infravermelho próximo do espectro eletromagnético, para detecção de infestação por fitonematóides.

\section{MATERIAL E MÉTODOS}

Foram tomadas medidas de espectrorradiometria de campo em área plantada com soja, variedade BRS 133, 112 dias após o plantio, naturalmente infestada por $H$. glycines.

O estudo foi conduzido no município de Florínea (lat. $22^{\circ}, 51^{\prime}$ S, long. 50, 39' W), no Vale do Paranapanema, tradicional região de cultivo de soja do Estado de São Paulo. O tipo de solo na área é latossolo vermelho escuro e o solo antes do preparo para plantio, apresentou $\mathrm{pH} 5,5$. Na adubação de pré-plantio foram utilizados $285 \mathrm{Kg} / \mathrm{ha}$ da formulação 0-20-20 e a lavoura foi plantada no dia 15 de outubro de 2000. A precipitação pluviométrica registrada do plantio até a tomada das leituras foi de $632 \mathrm{~mm}$, considerada normal em relação às médias anuais.

Para aquisição das leituras de espectrorradiometria, utilizou-se espectrorradiômetro portátil modelo SE590 (Spectron Inc., EUA) que obtém medidas de radiância na região espectral de 400 a $1.100 \mathrm{~nm}$ em 255 bandas espectrais. O aparelho consiste de unidade detectora (SE-390WB), ligada por cabos à unidade controladora (data-logger/CE-500) que, por sua vez, conecta-se a um microcomputador portátil do tipo "laptop", onde as medidas são armazenadas. Para as leituras, a unidade detectora foi fixada num mastro desmontável de alumínio, a cerca de $3 \mathrm{~m}$ sobre o dossel da cultura, utilizando-se uma lente com ângulo de visada de $15^{\circ}$, que permite obter radiância de superfície de aproximadamente $0,5 \mathrm{~m}^{2}$ (Figura 1). Juntamente ao mastro de fixação da unidade detectora, foi acoplada uma câmara fotográfica de $35 \mathrm{~mm}$ para permitir registro fotográfico do alvo nas mesmas condições de obtenção da medida espectrorradiométrica. As medidas foram tomadas entre 10:00 e 14:00 h, em condições de céu parcialmente aberto. $\mathrm{O}$ espectrorradiômetro foi posicionado verticalmente sobre as áreas amostrais no terreno. Em cada ponto amostrado, o equipamento foi rotacionado em torno do seu próprio eixo, de modo a permitir três 

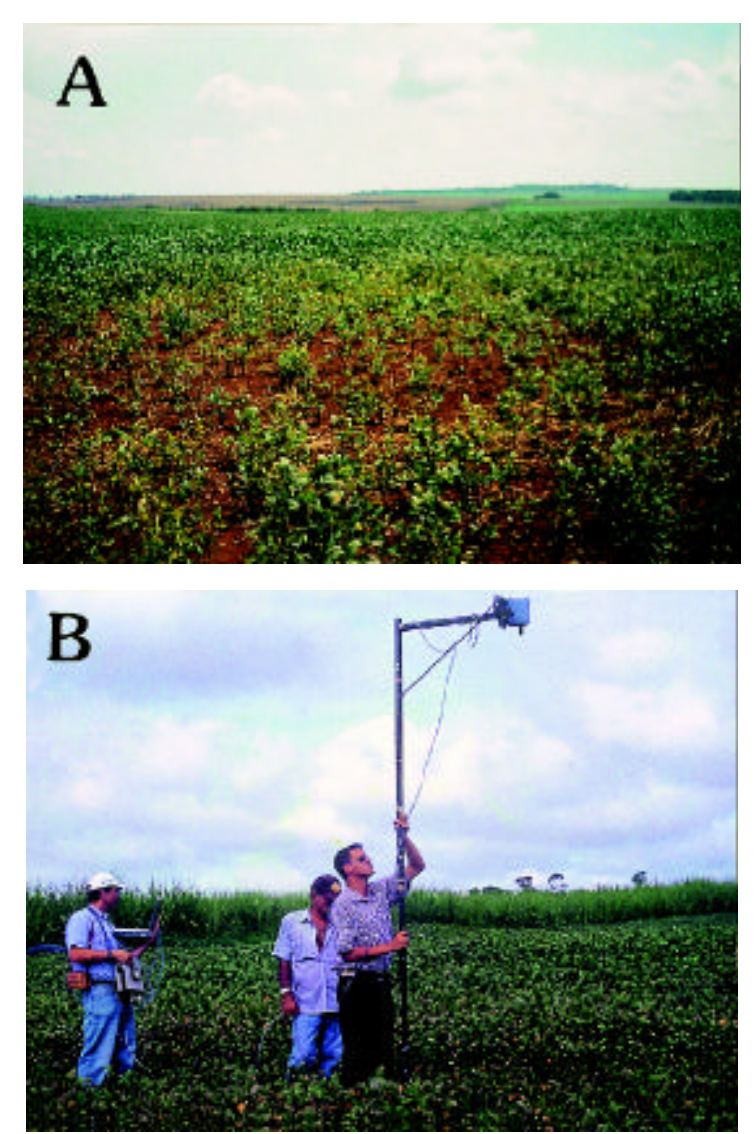

FIG. 1 - Utilização de medidas de espectrorradiometria, em uma área infestada por Heterodera glycines, plantada com soja (Glycine max) (variedade BRS133), 112 dias após o plantio. Em 1A observase aspecto da lavoura exibindo reboleira característica, formada a partir do ataque do nematóide. Em 1B, aquisição das medidas utilizando espectrorradiômetro portátil (Modelo SE590).

repetições das leituras em cada reboleira. As medidas do alvo foram intercaladas com medidas de radiância de uma placa de referência de sulfato de bário $\left(\mathrm{BaSO}_{4}\right)$. Este procedimento se fez necessário para que as medidas de radiância fossem transformadas em fator de reflectância.

As medidas espectrorradiométricas foram obtidas sobre 21 áreas amostrais, selecionadas por meio de avaliação visual, considerando níveis diferentes de sintomas de ataque do nematóide refletidos na lavoura. Os sintomas variaram de severo até ausência de sintomas. Cada área amostral foi georreferenciada utilizando-se receptor de GPS (Global Positioning System) de navegação, modelo Garmim GPS II. $\mathrm{O}$ georreferenciamento das amostras foi realizado com a finalidade de possibilitar, se necessário, o retorno à área ou para localização em imagem de sensoriamento remoto para posterior análise.

Os espectros obtidos foram processados em computador, utilizando-se o programa Espectro (Steffen et al., 1992). Este aplicativo calibrou os espectros de radiância das amostras com relação aos espectros de radiância da placa de referência, conforme mencionado anteriormente, e calculou os valores do fator de reflectância para as bandas espectrais correspondentes ao sensor TM do satélite Landsat, na banda TM3 (vermelho; 630 a $690 \mathrm{~nm}$ ), e na banda TM4 (infravermelho próximo; 760 a $900 \mathrm{~nm}$ ). Esses valores foram utilizados para o cálculo do NDVI de acordo com Tucker (1979), por meio da equação: NDVI = (TM4 - TM3) / (TM4 + TM3), onde a banda do TM4 corresponde ao pico da reflectância da cultura na região do infravermelho próximo e a banda TM3 corresponde ao pico da absortância da cultura na região do visível (vermelho). O NDVI expressa de forma combinada a resposta espectral das plantas nas regiões do vermelho (TM3) e infravermelho (TM4).

Em cada parcela onde foram tomadas leituras, coletouse com auxílio de enxadão, uma amostra composta de solo, a partir de três subamostras, retiradas numa profundidade de até $30 \mathrm{~cm}$. Essas amostras foram encaminhadas ao Laboratório de Nematologia do Departamento de Fitossanidade da UNESP/FCAV, Campus de Jaboticabal, SP, onde foram processadas segundo Jones (1945). Para cada amostra da área infestada por $\mathrm{H}$. glycines, foi determinado o número de cistos extraídos por $50 \mathrm{~cm}^{3}$ de solo.

Foram coletadas também cinco plantas de soja em cada área amostral, para determinação do peso da matéria fresca (PMF) e contagem do número de vagens por planta. Esses dados foram utilizados de forma complementar, para serem relacionados aos valores de NDVI e à infestação de nematóides.

\section{RESULTADOS E DISCUSSÃO}

De acordo com a resposta espectral das parcelas com níveis diferentes de infestação de $H$. glycines (Figura 2), na região do verde $(550 \mathrm{~nm})$, as curvas apresentaram um pequeno pico de reflexão, que confere a coloração verde da cultura. Na região do vermelho $(670 \mathrm{~nm})$, apenas uma pequena porção da energia incidente sobre as folhas foi refletida, uma vez que grande parte foi absorvida pelos pigmentos, para ser utilizada na fotossíntese. As plantas com menor infecção por nematóides, têm maior capacidade de absorção da energia incidente e, consequentemente, refletem menos no comprimento de onda centrado em $670 \mathrm{~nm}$ (vermelho). Na região do infravermelho próximo (a partir de $760 \mathrm{~nm}$ ) grande parte da energia incidente foi refletida devido á estrutura interna e número de camadas de folhas. As plantas, ou amostras situadas em pontos de menor densidade populacional de nematóides, apresentaram tendência de aumento nos valores do fator de reflectância (energia refletida) na região do infravermelho próximo, em função da maior quantidade de biomassa, em resposta ao menor ataque dos nematóides .

Os valores do NDVI (Tabela 1), da densidade da população de nematóides no solo, peso de matéria fresca e número de vagens obtidos para cada parcela da área infestada por $H$. glycines. Com os dados foram estabelecidas relações entre os parâmetros estudados. 


\section{R.F. Santos Júnior et al.}

TABELA 1 - Densidade populacional de Heterodera glycines por $50 \mathrm{~cm}^{3}$ de solo, peso da matéria fresca (PMF) em gramas, número de vagens por planta e índice vegetativo com diferença normalizada (NDVI), coletados em 21 áreas amostrais, em lavouras de soja (Glycine max) no Município de Florínea, SP

\begin{tabular}{ccccccc}
\hline \hline Amostra & Latitude & Longitude & $\begin{array}{c}\text { Cistos viáveis/ } \\
\mathbf{5 0}^{\mathbf{3}} \mathbf{\text { de solo }}\end{array}$ & PMF & $\begin{array}{c}\text { Vagens/ } \\
\text { Planta }\end{array}$ & NDVI \\
\hline 04 & $22^{\circ} 51^{\prime} 44,9^{\prime \prime}$ & $50^{\circ} 39^{\prime} 22,4^{\prime \prime}$ & 0 & 122,30 & 71 & 0,821 \\
16 & $22^{\circ} 50^{\prime} 59,8^{\prime \prime}$ & $50^{\circ} 39^{\prime} 11,0^{\prime \prime}$ & 0 & 158,10 & 63 & 0,881 \\
01 & $22^{\circ} 51^{\prime} 45,2^{\prime \prime}$ & $50^{\circ} 39^{\prime} 23,5^{\prime \prime}$ & 1 & 141,60 & 84 & 0,841 \\
12 & $22^{\circ} 50^{\prime} 59,8^{\prime \prime}$ & $50^{\circ} 39^{\prime} 10,1^{\prime \prime}$ & 2 & 66,00 & 30 & 0,494 \\
13 & $22^{\circ} 50^{\prime} 59,9^{\prime \prime}$ & $50^{\circ} 39^{\prime} 10,3^{\prime \prime}$ & 2 & 49,20 & 28 & 0,493 \\
09 & $22^{\circ} 51^{\prime} 43,3^{\prime \prime}$ & $50^{\circ} 39^{\prime} 23,7^{\prime \prime}$ & 2 & 145,20 & 65 & 0,827 \\
17 & $22^{\circ} 51^{\prime} 00,0^{\prime \prime}$ & $50^{\circ} 39^{\prime} 11,3^{\prime \prime}$ & 3 & 121,00 & 59 & 0,826 \\
10 & $22^{\circ} 51^{\prime} 31,4^{\prime \prime}$ & $50^{\circ} 39^{\prime} 10,4^{\prime \prime}$ & 3 & 160,50 & 69 & 0,889 \\
15 & $22^{\circ} 50^{\prime} 59,7^{\prime \prime}$ & $50^{\circ} 39^{\prime} 10,9 "$ & 3 & 156,50 & 76 & 0,896 \\
20 & $22^{\circ} 51^{\prime} 00,7^{\prime \prime}$ & $50^{\circ} 39^{\prime} 11,3^{\prime \prime}$ & 3 & 89,30 & 51 & 0,707 \\
14 & $22^{\circ} 51^{\prime} 00,2^{\prime \prime}$ & $50^{\circ} 39^{\prime} 10,2^{\prime \prime}$ & 4 & 104,10 & 67 & 0,815 \\
19 & $22^{\circ} 51^{\prime} 00,7^{\prime \prime}$ & $50^{\circ} 39^{\prime} 11,1^{\prime \prime}$ & 6 & 86,30 & 46 & 0,592 \\
08 & $22^{\circ} 51^{\prime} 44,0^{\prime \prime}$ & $50^{\circ} 39^{\prime} 22,7^{\prime \prime}$ & 7 & 64,80 & 54 & 0,734 \\
03 & $22^{\circ} 51^{\prime} 45,2^{\prime \prime}$ & $50^{\circ} 39^{\prime} 22,7^{\prime \prime}$ & 8 & 66,10 & 45 & 0,696 \\
07 & $22^{\circ} 51^{\prime} 43,8^{\prime \prime}$ & $50^{\circ} 39^{\prime} 22,6^{\prime \prime}$ & 8 & 61,10 & 34 & 0,598 \\
11 & $22^{\circ} 50^{\prime} 59,6^{\prime \prime}$ & $50^{\circ} 39^{\prime} 10,2^{\prime \prime}$ & 8 & 51,70 & 30 & 0,633 \\
02 & $22^{\circ} 51^{\prime} 45,1^{\prime \prime}$ & $50^{\circ} 39^{\prime} 23,2^{\prime \prime}$ & 9 & 73,60 & 24 & 0,542 \\
18 & $22^{\circ} 51^{\prime} 00,4^{\prime \prime}$ & $50^{\circ} 39^{\prime} 11,3^{\prime \prime}$ & 10 & 56,10 & 25 & 0,447 \\
05 & $22^{\circ} 51^{\prime} 44,5^{\prime \prime}$ & $50^{\circ} 39^{\prime} 22,3^{\prime \prime}$ & 12 & 33,06 & 22 & 0,308 \\
21 & $22^{\circ} 51^{\prime} 00,3^{\prime \prime}$ & $50^{\circ} 39^{\prime} 11,5^{\prime \prime}$ & 23 & 28,00 & 17 & 0,415 \\
06 & $22^{\circ} 51^{\prime} 44,5^{\prime \prime}$ & $50^{\circ} 39^{\prime} 22,4 "$ & 56 & 23,70 & 16 & 0,154 \\
\hline
\end{tabular}

A análise desses dados indicou que, em geral, a relação entre população de $H$. glycines $\left(\mathrm{N} .^{\circ}\right.$ de nematóides $/ 50 \mathrm{~cm}^{3} \mathrm{de}$ solo) e peso da matéria fresca (PMF), número de vagens/planta e NDVI é inversa. Essa relação não se aplica às amostras 12 e 13, as quais, com baixa densidade populacional de cistos (2 cistos $/ 50 \mathrm{~cm}^{3}$ de solo) apresentaram valores de NDVI de 0,494 e 0,493 respectivamente. $O$ mapa de coleta das amostras na área, indicou que as duas pertencem a locais contíguos, sugerindo que outros fatores poderiam estar envolvidos além do ataque de $H$. glycines. Alston et al. (1993) constataram efeito aditivo da interação entre o nematóide de cisto da soja e a competição da cultura com plantas daninhas e o ataque de pragas (Helicoverpa zea. Bod.) ocasionando, em média, o dobro dos danos causados pelo NCS isolado. De fato, problemas de drenagem e fertilidade do solo em pontos da lavoura, causam depauperamento das plantas e também podem agravar os sintomas de ataque dos nematóides. O peso de matéria verde e número de vagens exibiram uma relação direta com os valores de NDVI.

A relação entre dois parâmetros pode ser estimada pela correlação de Pearson (Seber, 1977), um fundamento da estatística utilizado como análise exploratória de um conjunto de dados (Lamparelli et al. 2001). Essa análise foi efetuada considerando-se a existência de estrutura de dependência entre os parâmetros envolvidos no sistema. Os coeficientes de correlação entre os critérios envolvidos na avaliação (Nematóides por $50 \mathrm{~cm}^{3}$, PMF, Vagens/Planta e NDVI) indicaram que estão altamente correlacionados nas áreas infestadas por $H$. glycines (Tabela 2 ).

Todos os parâmetros considerados nessa análise, apresentaram correlação altamente significava $(\mathrm{p}<0,01)$. A correlação amostral entre peso da matéria fresca e número de vagens, peso da matéria fresca e NDVI e número de vagens por planta e NDVI, foi de alta magnitude. A correlação amostral entre densidade populacional de $\mathrm{H}$. glycines por 50 $\mathrm{cm}^{3}$ de solo (Nematóides) e NDVI, também foi de alta magnitude, com a diferença de que, nesse caso, trata-se de correlação alta negativa. Uma alta correlação entre NDVI e parâmetros de produtividade, também foi constatada em estudos de radiometria de campo, conduzidos por Stafford \& Bolam (1996).

A influência do ataque de fitonematóides no

TABELA 2 - Coeficientes de correlação entre Densidade populacional de Heterodera glycines por 50 $\mathbf{c m}^{3}$ de solo (Nematóides), peso da matéria fresca (PMF) em gramas, número de vagens por planta e índice vegetativo com diferença normalizada (NDVI), obtidas em 21 áreas amostrais de $0,5 \mathrm{~m}^{2}$, em lavoura de soja (Glycine max) no município de Florínea, SP

\begin{tabular}{lcccc}
\hline \hline & PMF & Vagens/Planta & NDVI & Nematóides \\
\hline PMF & 1 & $0,9089 * *$ & $0,8897^{* *}$ & $-0,5835^{* *}$ \\
Vagens/Planta & & 1 & $0,9163^{* *}$ & $-0,5786^{* *}$ \\
NDVI & & & 1 & $-0,7350^{* *}$ \\
Nematóides & & & & 1 \\
\hline
\end{tabular}

* Significativo ao nível de 5\% ( $\mathrm{p}<0,05)$,

** Significativo ao nível de $1 \%(\mathrm{p}<0,01)$, NS: Não significativo $(p>0,05)$. 
Detecção de Heterodera glycines em plantios de soja mediante espectrorradiometria...
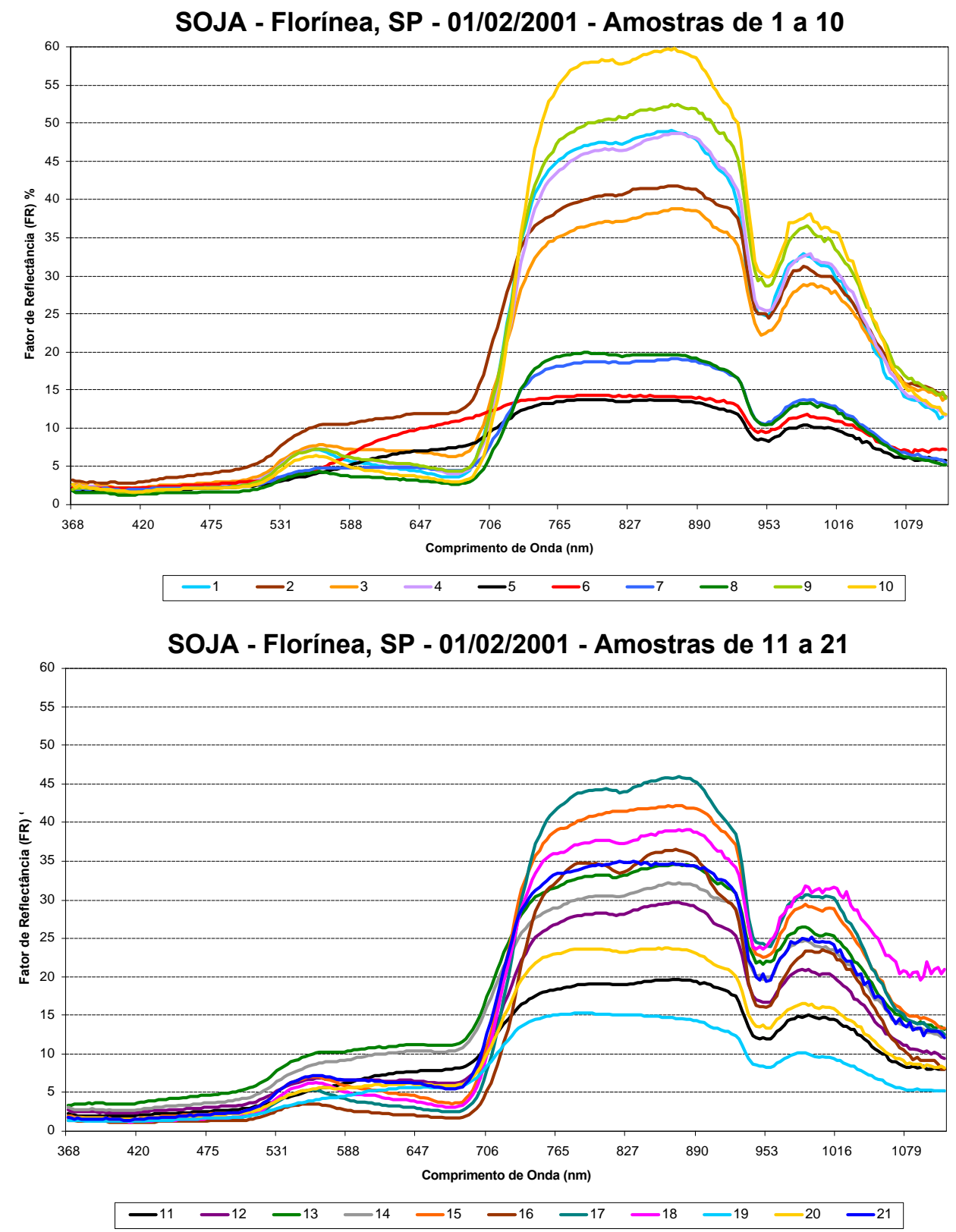

FIG. 2 - Resposta espectral de cultura de soja (Glycine Max) (variedade BRS133), 112 dias após o plantio, para diferentes níveis de infestação de Heterodera glycines, em lavoura comercial no Município de Florínea, SP.

comportamento espectral das plantas também foi observada por Gausmam et al. (1975). Esses autores efetuando medidas espectrorradiométricas de plantas de algodoeiro (Gossypium hirsutum L.), mantidas em vasos com diferentes níveis populacionais do nematóide reniforme (Rotylenchus reniformis Lindorf \& Oliveira), encontraram em plantas atacadas, respostas espectrais diferentes das obtidas em plantas saudáveis.

Os espectros apresentaram, de maneira lógica, a evolução dos valores de NDVI, em face dos sintomas de ataque dos nematóides, expressos na cultura e traduzidos por um volume de biomassa verde variável, em geral, de acordo com a infestação. Os resultados permitiram assumir que $H$. glycines influenciou a resposta espectral das plantas de soja. Por conseguinte, é possível detectar e mapear áreas de soja infestadas por esses nematóides utilizando-se técnicas de sensoriamento remoto.

\section{LITERATURA CITADA}

ALSTON, D.G., SHMITT, D.P., BRADLEY JUNIOR, J.R. \& COBLE, H.D. Multiple pest interactions in soybean: effects on Heterodera glycines egg populations and crop yield. Journal of Nematology 25:42-49. 1993.

AMARAL, S., KAZMIERCZAK, M.L., ZERBIANI, N.J. \& 


\section{R.F. Santos Júnior et al.}

CARVALHO, V.C. Comportamento espectral de mudas de Eucalyptus grandis (Hill) Maiden em diferentes percentagens de cobertura. INPE, São José dos Campos. 1990.

BATISTA, G.T. \& RUDORFF, B.F.T. Spectral response of soybean by field radiometry. Journal of Photogrammetry and Remote Sensing 45:111-121. 1990.

BRENCHLEY, G.H. Aerial photography for the study of plant diseases. Annual Review of Phytopathology 6:1-23. 1968.

DAUGHTRY, C.S.T., GALLO, K.P., BIEHL. L.L., KANEMASU, E.T., ASRAR, G., BLAD, B.L., NORMAN, J.M. \& GARDNER, B.R. Spectral estimatives of agronomic characteristics of crops. Machine Processing of Remote Sensed Data Symposium. Purddue University, W. Lafayette, IN, 1984.

FORMAGGIO, A.R. Características agronômicas e espectrais para sensoriamento remoto de trigo e de feijão. (Tese de Doutorado). Universidade Estadual de São Paulo. 1985

GAUSMAN, H.W., HEALD C.M. \& ESCOBAR, D.E. Efect of Rotylenchulus reniformis on refletance of cotton plant leaves. Journal of Nematology 7:368-374. 1975.

HOLBEN, B.N., TUCKER, C.J. \& FAN, C.J. Spectral assesment of soybean leaf area and leaf biomass. Photogrammetric Engineering \& Remote Sensing 46:651-656. 1980.

JONES, F.G.W. Soil populations of beet eelworm (Heterodera schachtii Schm.) in relation to cropping. Annals Applied Biology 32:351-380. 1945.

KNIPLING, E.B. Physical and physiological basis for the reflectance of visible and near-infrared radiation from vegetation. Remote Sensing of Environment 3:155-159. 1970.

LAMPARELLI, R.A.C., ROCHA, J.V. \& BORGHI, E., Geoprocessamento e agricultura de precisão. Guaíba. 2001.

MYERS, V.I. Soil, water and plant relations. In: National Academy of Scients. Remote Sensing with special reference to agricultural and forestry. Washington. 1970.

PESQUISA FAPESP. Expansão da soja prejudica o cerrado 62:2226. 2001

QI, J., Chehbouni, A., HUETE, A.R., KERR, Y.H. \& SOROOSHIAN, S. A modified soil adjusted vegetation index. Remote Sensing Enviromental 48:119-126. 1994.

RUDORFF, B.F.T., MOREIRA, M.A., OVIEDO, A. \& FREITAS, J.G. Efeito do nitrogênio e do déficit hídrico na resposta espectral de cultivares de trigo. Anais, Simposio Latino Americano de Percepción Remota. Mérida, Venezuela. CD-ROM. 1997.

SEBER, G.A.S. Linear regression analysis. New York. John Willey \& Sons. 1977.

STAFFORD, J.V.\& BOLAM, H.C. Inproving reliability of position resolution using GPS for precision agriculture. Abstract, International Conference On Agricultural Engineering, Madri 2:985-986. 1996

STEFFEN, C.A., OLIVEIRA de, J.L. \& GAMA, F.F. O programa Espectro. NT LARAD. 1992.

TAYLOR, J.C., THOMAS, G. \& WOOD, G.A. Mapping yield potential with remote sensing. Anais. Precision Agriculture'97 2:713-72. 1997.

TUCKER, C.J. Red and photographic infrared linear combination for monitoring vegetation. Remote Sensing of Environment. 8:127-50. 1979.

VALERIANO, M.M. Reflectância espectral de trigo irrigado (Triticum aestivum L.) por espectrorradiometria de campo. (Dissertação de Mestrado). São José dos Campos. Instituto Nacional de Pesquisas Espaciais. 1992.

YORINORI, J.T. Soja (Glycine max (L.) Merril) controle de doenças. In: Vale, F.X.R., Zambolim, L. (Eds.). Controle de doenças de plantas. Viçosa: Universidade Federal de Viçosa. 1997. pp.9531009. 